Article

\title{
Mean Values of Products of $L$-Functions and Bernoulli Polynomials
}

\author{
Abdelmejid Bayad ${ }^{1}$ and Daeyeoul Kim ${ }^{2, *}$ \\ 1 Laboratoire de Mathematiques et Modélisation d'Évry (UMR 8071), Université d'Évry Val d'Essonne, \\ Université Paris-Saclay, I.B.G.B.I., 23 Bd. de France, 91037 Évry CEDEX, France; abayad@maths.univ-evry.fr \\ 2 Department of Mathematics, Institute of Pure and Applied Mathematics, Chonbuk National University, \\ 567 Baekje-daero, Deokjin-gu, Jeonju-si 54896, Korea \\ * Correspondence: kdaeyeoul@jbnu.ac.kr
}

Received: 17 November 2018; Accepted: 14 December 2018; Published: 19 December 2018

Abstract: Let $m_{1}, \cdots, m_{r}$ be nonnegative integers, and set:

$$
M_{r}=m_{1}+\cdots+m_{r}
$$

In this paper, first we establish an explicit linear decomposition of:

$$
\prod_{i=1}^{r} \frac{B_{m_{i}}(x)}{m_{i} !}
$$

in terms of Bernoulli polynomials $B_{k}(x)$ with $0 \leq k \leq M_{r}$. Second, for any integer $q \geq 2$, we study the mean values of the Dirichlet $L$-functions at negative integers:

$$
\sum_{\chi_{1}, \cdots, \chi_{r}(\bmod q) ; \chi_{1} \cdots \chi_{r}=1} \prod_{i=1}^{r} L\left(-m_{i}, \chi_{i}\right)
$$

where the summation is over Dirichlet characters $\chi_{i}$ modulo $q$. Incidentally, a part of our work recovers Nielsen's theorem, Nörlund's formula, and its generalization by $\mathrm{Hu}$, Kim, and Kim.

Keywords: Dirichlet character; Bernoulli polynomials; mean value of the $L$-function

MSC: 11B68; 11M06.

\section{Notations and Introduction}

As usual, we define the Bernoulli polynomials $B_{n}(x)$ through the generating function:

$$
\frac{t}{e^{t}-1} e^{x t}=\sum_{n \geq 0} B_{n}(x) \frac{t^{n}}{n !}
$$

and the Bernoulli numbers are given by $B_{n}=B_{n}(0)$. Moreover, from (1), we have:

$$
B_{2 k+1}=0 \text { for all } k \geqslant 1 \text {, and } B_{1}=-1 / 2 \text {. }
$$

Let $q$ be a positive integer, we denote by $\chi$ a Dirichlet character modulo $q$, and $L(s, \chi)$ a Dirichlet $L$-series is given by:

$$
L(s, \chi)=\sum_{n \geq 1} \frac{\chi(n)}{n^{s}}
$$


for $\operatorname{Re}(s)>0$ if $\chi$ is non-principal and $\operatorname{Re}(s)>1$ if $\chi$ is the principal character modulo $q$.

For a Dirichlet character $\chi$ modulo $q$, the generalized Bernoulli numbers:

$$
B_{n, \chi} \in \mathbb{Q}(\chi(1), \chi(2), \ldots, \chi(q-1))
$$

are given through the generating function:

$$
\sum_{a=1}^{q} \chi(a) \frac{t e^{a t}}{e^{q t}-1}=\sum_{n=0}^{\infty} B_{n, x} \frac{t^{n}}{n !}
$$

Therefore, we have:

$$
B_{n, \chi}=q^{n-1} \sum_{a=1}^{q-1} \chi(a) B_{n}(a / q) .
$$

The main interest of the numbers $B_{n, \chi}$ is that they give the value at non-positive integers of Dirichlet $L$-series. In fact, there is a well-known formula proven by Hecke in [1]:

$$
L(1-n, \chi)=-\frac{B_{n, \chi}}{n}(n \geq 1) .
$$

We are motivated by the arithmetic properties satisfied by the finite product of several generalized Bernoulli numbers $B_{n, \chi}$ and also the product of Bernoulli polynomials.

Nielsen gave the following important result for the product of two Bernoulli polynomials.

Theorem 1 ([2], p. 75). For $m_{1}, m_{2} \geq 1$, we have the formula:

$$
\begin{gathered}
\frac{B_{m_{1}}(x)}{m_{1} !} \frac{B_{m_{2}}(x)}{m_{2} !}=(-1)^{m_{2}-1} \frac{B_{m_{1}+m_{2}}}{\left(m_{1}+m_{2}\right) !}+ \\
\sum_{k=0}^{\left[\left(m_{1}+m_{2}-1\right) / 2\right]}\left[\left(\begin{array}{c}
m_{1}+m_{2}-1-2 k \\
m_{1}-1
\end{array}\right)+\left(\begin{array}{c}
m_{1}+m_{2}-1-2 k \\
m_{2}-1
\end{array}\right)\right] \frac{B_{2 k}}{2 k !} \frac{B_{m_{1}+m_{2}-2 k}(x)}{\left(m_{1}+m_{2}-2 k\right) !} .
\end{gathered}
$$

At the same time, Nörlund [3] gave formulas for the integral of the product of two Bernoulli polynomials.

Theorem 2 ([3], p. 31). For $m_{1}, m_{2} \geq 1$, we have the formula:

$$
\int_{0}^{1} \frac{B_{m_{1}}(x)}{m_{1} !} \frac{B_{m_{2}}(x)}{m_{2} !} d x=(-1)^{m_{2}-1} \frac{B_{m_{1}+m_{2}}}{\left(m_{1}+m_{2}\right) !} .
$$

Note that Theorem 2 can be obtained directly from Theorem 1 . However, the proofs of Nörlund and Nielsen are different.

We should mentioned that later, Mordell [4] and Mikolás [5] provided two other different proofs of (7).

Recently, D. Zagier ([6], pp. 250-252) revisited the results of Nielsen (Theorem 1) and Nörlund (Theorem 2) and gave another proof.

Carlitz [7] studied the integrals of the product of three and four Bernoulli polynomials. Furthermore, the results by Carlitz-Mikolas-Mordell-Nörlund were generalized by $\mathrm{Hu}$, Kim, and Kim in [8] as follows. 
Theorem 3 ([8]). Let $m_{1}, \ldots, m_{d} \geq 1, d \geq 2$ be integer, we have the formula:

$$
\begin{aligned}
& \int_{0}^{1} \prod_{i=1}^{d} \frac{B_{m_{i}}(x)}{m_{i} !} d x= \\
& \sum_{k=0}^{M_{d-1}}(-1)^{k} \sum_{k_{1}+\cdots+k_{d-1}=k}\left(\begin{array}{c}
k \\
k_{1}, \cdots, k_{d-1}
\end{array}\right) C\left(m_{1}-k_{1}, \cdots, m_{d-1}-k_{d-1}, m_{d}+k+1\right),
\end{aligned}
$$

where:

$$
C\left(k_{1}, \cdots, k_{d}\right)=\frac{1}{k_{1} ! \cdots k_{d} !}\left(B_{k_{1}}(1) \cdots B_{k_{d}}(1)-B_{k_{1}} \cdots B_{k_{d}}\right)
$$

and $M_{d-1}=m_{1}+\cdots+m_{d-1}$, and $\left(\begin{array}{c}k \\ k_{1}, \cdots, k_{d-1}\end{array}\right)$ is the multinomial coefficients.

Similar integral evaluations have also been studied by Espinosa and Moll [9-11]. The purpose of this paper is to prove a generalization of Nielsen's Theorem 1 and to study the mean values of $L$-functions at negative integers and their connections to Bernoulli-Dedekind sums.

We consider:

$$
\phi_{m_{1}, \ldots, m_{d}}(x):=\frac{B_{m_{1}}(x)}{m_{1} !} \cdots \frac{B_{m_{d}}(x)}{m_{d} !}
$$

$\phi_{m_{1}, \ldots, m_{d}}(x)$ is a polynomial in $x$ with degree $M_{d}=m_{1}+\cdots+m_{d}$, since $\frac{B_{k}(x)}{k !}$ is a polynomial of degree $k$ in $x$. Then, $\left\{1, B_{1}(x), \frac{B_{2}(x)}{2 !}, \ldots, \frac{B_{M_{d}}(x)}{M_{d} !}\right\}$ is a basis of the polynomial space $\mathbb{R}_{M_{d}}[x]$ over $\mathbb{R}$. There exist real numbers $a_{k}\left(m_{1}, \ldots, m_{d}\right), 0 \leq k \leq M_{d}$, such that:

$$
\phi_{m_{1}, \ldots, m_{d}}(x):=\sum_{k=0}^{M_{d}} a_{k}\left(m_{1}, \ldots, m_{d}\right) \frac{B_{k}(x)}{k !} .
$$

As the first goal of this paper, we prove explicit formulas for the coefficients $a_{k}\left(m_{1}, \ldots, m_{d}\right)$ with $0 \leq k \leq M_{d}$. In the second part of this paper, we establish relationships between these coefficients and mean values of Dirichlet $L$-series at negative integers.

\section{Statement of the Main Results}

Now, we state our main results.

Theorem 4. Let $m_{1}, \ldots, m_{d} \geqslant 1$ be positive integers. We have the explicit formula:

$$
\begin{gathered}
\prod_{i=1}^{d} \frac{B_{m_{i}}(x)}{m_{i} !}= \\
\left((-1)^{M_{d}+1}-1\right) \sum_{k_{d}=0}^{M_{d-1}}(-1)^{k_{d}} \sum_{k_{1}+\cdots+k_{d-1}=k_{d}}\left(\begin{array}{c}
k_{d} \\
k_{1}, \ldots, k_{d-1}
\end{array}\right)\left(\prod_{i=1}^{d-1} \frac{B_{m_{i}-k_{i}}}{\left(m_{i}-k_{i}\right) !}\right) \frac{B_{m_{d}+k_{d}+1}}{\left(m_{d}+k_{d}+1\right) !} \\
+\sum_{1 \leq l \text { odd } \leq M_{d}} a_{M_{d}-l+1}\left(m_{1}, \ldots, m_{d}\right) \frac{B_{M_{d}-l+1}(x)}{\left(M_{d}-l+1\right) !}
\end{gathered}
$$


where:

$$
a_{M_{d}-l+1}\left(m_{1}, \ldots, m_{d}\right)=\sum_{0 \leq k \text { odd } \leq d} \frac{1}{2^{k-1}} \sum_{\substack{|I|=k \\
0 \leq k_{i} \text { even } \leq m_{i} \\
\sum_{i \in \bar{I}} k_{i}=l-k}}\left(\begin{array}{c}
M_{d}-l \\
m_{j}-1, m_{j}-k_{j} \\
(j \in I)
\end{array}\right)\left(\prod_{\substack{(j \in \bar{I}) \\
i \in \bar{I}}} \frac{B_{k_{i}}}{k_{i} !}\right)
$$

with $M_{d}=m_{1}+\cdots+m_{d}$ and $M_{d-1}=m_{1}+\cdots+m_{d-1}$. Here, $I \subset\{1,2, \cdots, d\}, I \cap \bar{I}=\varnothing$, and $I \cup \bar{I}=\{1,2, \cdots, d\}$.

We have our second main result.

Theorem 5. Let $q \geq 2, m_{1}, \ldots, m_{d} \geq 0$ be nonnegative integers and $d \geq 2$. We have the mean values:

$$
\sum_{\substack{\chi_{1}, \cdots, \chi_{d}(\bmod q) \\ x_{1} \cdots \chi_{d}=1}} \prod_{i=1}^{d} L\left(-m_{i}, \chi_{i}\right)=(-1)^{d} q^{M_{d}} m_{1} ! \cdots m_{d} ! \varphi(q)^{d-1} S\left(m_{1}, \ldots, m_{d} ; q\right)
$$

with:

$$
S\left(m_{1}, \ldots, m_{d} ; q\right)=\sum_{j=0}^{M_{d}+d}(-1)^{j} a_{j}\left(m_{1}+1, \ldots, m_{d}+1\right) \frac{B_{j}}{j !} J_{1-j}(q),
$$

where $\varphi$ is the Jordan totient function, $J_{k}(q)=q^{k} \prod_{p \text { prime|q }}\left(1-p^{-k}\right)$ is the $k^{\text {th }}$ Jordan function, the summation is over all Dirichlet characters $\chi_{1}, \cdots, \chi_{d}$ modulo $q$, and the coefficients $a_{j}$ are given by Formula (9).

Theorem 5 can be viewed as a complement to the recent work of Bayad-Raouj [12] on the Mean values of $L$-functions at positive integers.

We can restate Theorem 5 in terms of the generalized Bernoulli numbers $B_{n, \chi}$ as follows.

Theorem 6. Let $q \geq 2, m_{1}, \ldots, m_{d} \geq 0$ be non-negative integers and $d \geq 2$. We have the mean values:

$$
\sum_{\substack{\chi_{1}, \cdots, \chi_{d}(\bmod q) \\ \chi_{1} \cdots \chi_{d}=1}} \prod_{i=1}^{d} \frac{B_{m_{i}+1, \chi_{i}}}{\left(m_{i}+1\right) !}=q^{M_{d}} \varphi(q)^{d-1} S\left(m_{1}, \ldots, m_{d} ; q\right) .
$$

Let us state some special cases of Theorem 5 .

Corollary $\mathbf{1}(d=2)$. Let $q \geq 2, m, n$ be nonnegative integers. We have the mean values:

$$
\begin{gathered}
\sum_{\chi(\bmod q)} L(-m, \chi) L(-n, \bar{\chi})=q^{m+n} \varphi(q)^{2}(-1)^{n} \frac{B_{m+n+2}}{(m+n+2) !} \\
+(-1)^{m+n} q^{m+n} \varphi(q) \sum_{k=0}^{[m+n+1 / 2]}\left[\left(\begin{array}{c}
m+n+1-2 k \\
m
\end{array}\right)+\left(\begin{array}{c}
m+n+1-2 k \\
n
\end{array}\right)\right] \\
\times \frac{B_{2 k} B_{m+n+2-2 k}}{(2 k) !(m+n+2-2 k) !} J_{2 k-m-n-1}(q) .
\end{gathered}
$$


Corollary $2(d=3)$. Let $q \geq 2, m_{1}, m_{2}, m_{3}$ be nonnegative integers. We have the mean values:

$$
\begin{gathered}
\sum_{\chi_{1}, \chi_{2}} L\left(-m_{1}, \chi_{1}\right) L\left(-m_{2}, \chi_{2}\right) L\left(-m_{3}, \overline{\chi_{1} \chi_{2}}\right)= \\
(-1)^{m_{3}} q^{m_{1}+m_{2}+m_{3}} \varphi(q)^{3} m_{1} ! m_{2} ! m_{3} ! \sum_{k=0}^{m_{1}+m_{2}+2}\left\{\left(\begin{array}{c}
k \\
m_{1}
\end{array}\right)+\left(\begin{array}{c}
k \\
m_{2}
\end{array}\right)\right\} \frac{B_{m_{3}+k+2}}{\left(m_{3}+k+2\right) !} \frac{B_{m_{1}+m_{2}+1-k}}{\left(m_{1}+m_{2}+1-k\right) !}+ \\
q^{m_{1}+m_{2}+m_{3}} \varphi(q)^{2} m_{1} ! m_{2} ! m_{3} ! \sum_{k_{1}, k_{2} \text { even }} c_{k_{1}, k_{2}} \frac{B_{k_{1}} B_{k_{2}} B_{m_{1}+m_{2}+m_{3}+2-k_{1}-k_{2}}}{k_{1} ! k_{2} !\left(m_{1}+m_{2}+m_{3}+2-k_{1}-k_{2}\right) !} J_{-m_{1}-m_{2}-m_{3}-1+k_{1}+k_{2}} \\
(q)+q^{m_{1}+m_{2}+m_{3}} \varphi(q)^{2} \frac{m_{1} ! m_{2} ! m_{3} !}{4}\left(\begin{array}{c}
m_{1}+m_{2}+m_{3} \\
m_{1}, m_{2}, m_{3}
\end{array}\right) \frac{B_{m_{1}+m_{2}+m_{3}+1}}{\left(m_{1}+m_{2}+m_{3}+1\right) !} J_{-m_{1}-m_{2}-m_{3}(q),}
\end{gathered}
$$

where:

$$
c_{k_{1}, k_{2}}=\left(\begin{array}{l}
m_{1}+m_{2}+m_{3}+2-k_{1}-k_{2} \\
m_{1}, m_{2}+1-k_{1}, m_{3}+1-k_{2}
\end{array}\right)+\left(\begin{array}{l}
m_{1}+m_{2}+m_{3}+2-k_{1}-k_{2} \\
m_{1}+1-k_{1}, m_{2}, m_{3}+1-k_{2}
\end{array}\right)+\left(\begin{array}{l}
m_{1}+m_{2}+m_{3}+2-k_{1}-k_{2} \\
m_{1}+1-k_{1}, m_{2}+1-k_{2}, m_{3}
\end{array}\right) .
$$

\section{Proof of Theorem 4}

We start this section with some useful lemmas.

\subsection{Three Lemmas}

Let $m_{1}, \ldots, m_{d}$ be non-negative integers and:

$$
M_{d}:=m_{1}+\cdots+m_{d}>1 \text {. }
$$

From Equation (1), we obtain the following results.

Lemma 1. For $n \geq 1$, we have:

$$
\begin{aligned}
& \frac{B_{n}(x+1)}{n !}-\frac{B_{n}(x)}{n !}=\frac{x^{n-1}}{(n-1) !} \\
& \frac{B_{n}(x+y)}{n !}=\sum_{k=0}^{n} \frac{B_{k}(y)}{k !} \frac{x^{n-k}}{(n-k) !}
\end{aligned}
$$

Let:

$$
f_{n}(x):=\sum_{0 \leq k \text { even } \leq n} \frac{B_{k}}{k !} \frac{x^{n-k}}{(n-k) !}=\frac{B_{n}(x)}{n !}+\frac{1}{2} \frac{x^{n-1}}{(n-1) !} .
$$

Write:

$$
\Delta \phi(x):=\phi(x+1)-\phi(x)
$$

We have:

$$
\Delta \phi_{m_{1}, \ldots, m_{d}}(x)=\sum_{k=0}^{M_{d}} a_{k}\left(m_{1}, \ldots, m_{d}\right) \Delta\left(\frac{B_{k}(x)}{k !}\right)=\sum_{k=1}^{M_{d}} a_{k}\left(m_{1}, \ldots, m_{d}\right) \frac{x^{k-1}}{(k-1) !} .
$$

Then, we get:

$$
\Delta \phi_{m_{1}, \ldots, m_{d}}(x)=\sum_{k=0}^{M_{d}-1} a_{k+1}\left(m_{1}, \ldots, m_{d}\right) \frac{x^{k}}{k !}
$$


and:

$$
\phi_{m_{1}, \ldots, m_{d}}(x)=\prod_{i=1}^{d} \frac{B_{m_{i}}(x)}{m_{i} !}=\prod_{i=1}^{d}\left(f_{m_{i}}(x)-\frac{1}{2} \frac{x^{m_{i}-1}}{\left(m_{i}-1\right) !}\right) .
$$

Now, we recall the general polynomial multiplication formulas.

Lemma 2. Let $Q_{i}(x)=\sum_{k_{i}=0}^{m_{i}} a_{k_{i}}^{(i)} x^{k_{i}}$. Then, we have:

$$
\prod_{i=1}^{d} Q_{i}(x)=\sum_{\substack{0 \leq k_{1} \leq m_{1} \\ 0 \leq k_{d} \leq m_{d}}} a_{k_{1}}^{(1)} \cdots a_{k_{d}}^{(d)} x^{k_{1}+\cdots+k_{d}}=\sum_{k=0}^{M_{d}}\left(\sum_{\substack{0 \leq k_{i} \leq m_{i}, i=1, \ldots, d \\ k_{1}+\ldots+k_{d}=k}} a_{k_{1}}^{(1)} \cdots a_{k_{d}}^{(d)}\right) x^{k} .
$$

Lemma 3. Let $d$ be a positive integer. Then, we have:

$$
\prod_{i=1}^{d}\left(x_{i}+a_{i}\right)=\sum_{I \subset\{1, \ldots, d\}}\left(\prod_{i \in I} a_{i}\right)\left(\prod_{i \in \bar{I}} x_{i}\right) .
$$

\subsection{Proof of Theorem 4}

By Lemmas 1, 2, and 3, we have:

$$
\phi_{m_{1}, \ldots, m_{d}}(x)=\prod_{i=1}^{d}\left(f_{m_{i}}(x)-\frac{1}{2} \frac{x^{m_{i}-1}}{\left(m_{i}-1\right) !}\right)=\sum_{I \subset\{1, \ldots, d\}}\left(-\frac{1}{2}\right)^{\# I} \frac{x^{M_{I}-|I|}}{\prod_{i \in I}\left(m_{i}-1\right) !} \prod_{i \in \bar{I}} f_{m_{i}}(x),
$$

where $M_{I}=\sum_{i \in I} m_{i}$ and $\bar{I}=\{1, \cdots, d\} \backslash I$. Then, we get:

$$
\Delta \phi_{m_{1}, \ldots, m_{d}}(x)=\sum_{\substack{I \subset\{1, \ldots, d\}, \# I \text { odd }}} \frac{1}{2^{\# I-1}} \frac{x^{M_{I}-|I|}}{\prod_{i \in I}\left(m_{i}-1\right) !} \prod_{i \in \bar{I}} f_{m_{i}}(x) .
$$

On the other hand, we consider:

$$
\prod_{i=1}^{d} f_{m_{i}}(x)=\prod_{i=1}^{d}\left(\frac{B_{m_{i}}(x)}{m_{i} !}+\frac{1}{2} \frac{x^{m_{i}-1}}{\left(m_{i}-1\right) !}\right)=\prod_{i=1}^{d}\left(\sum_{0 \leq k_{i} \text { even } \leq m_{i}} \frac{B_{k_{i}}}{k_{i} !} \frac{x^{m_{i}-k_{i}}}{\left(m_{i}-k_{i}\right) !}\right) .
$$

Therefore, we obtain:

$$
\prod_{i=1}^{d} f_{m_{i}}(x)=\sum_{\substack{0 \leq k_{i} \text { even } \leq m_{i} \\ i=1, \ldots, d}}\left(\prod_{i=1}^{d} \frac{B_{k_{i}}}{k_{i} !}\right) \frac{x^{M_{d}-\sum_{i=1}^{d} k_{i}}}{\prod_{i=1}^{d}\left(m_{i}-k_{i}\right) !}
$$

By Lemma 2, we have:

$$
\prod_{i=1}^{d} f_{m_{i}}(x)=\sum_{k=0}^{M_{d}}\left(\sum_{\substack{0 \leq k_{i} \text { even } \leq m_{i} \\
i=1, \ldots, d \\
k_{1}+\ldots+k_{d}=k}}\left(\begin{array}{c}
M_{d}-k \\
m_{1}-k_{1}, \ldots, m_{d}-k_{d}
\end{array}\right) \prod_{i=1}^{d} \frac{B_{k_{i}}}{k_{i} !}\right) \frac{x^{M_{d}-k}}{\left(M_{d}-k\right) !} .
$$


Hence,

$$
\Delta \phi_{m_{1}, \ldots, m_{d}}(x)=\sum_{0 \leq k \text { odd } \leq d} \frac{1}{2^{k-1}} \sum_{|I|=k} \frac{x^{M_{I}-k}}{\prod_{i \in I}\left(m_{i}-1\right) !} \prod_{i \in \bar{I}} f_{m_{i}}(x)
$$

and:

$$
\prod_{i \in \bar{I}} f_{m_{i}}(x)=\sum_{t=0}^{M_{\bar{I}}}\left(\sum_{\substack{\sum_{i} \in \bar{I}_{i}=t \\
0 \leq k_{i} \text { even } \leq m_{i}}}\left(\begin{array}{c}
M_{\bar{I}}-k \\
m_{i}-k_{i}, i \in \bar{I}
\end{array}\right) \prod_{i \in \bar{I}} \frac{B_{k_{i}}}{k_{i} !}\right) \frac{x^{M_{\bar{I}}-t}}{\left(M_{\bar{I}}-t\right) !}
$$

Thus, we get:

$$
\Delta \phi_{m_{1}, \ldots, m_{d}}(x)=\sum_{0 \leq k \text { odd } \leq d} \frac{1}{2^{k-1}} \sum_{\substack{|I|=k \\
0 \leq t \text { even } \leq M_{\bar{I}}}} \sum_{\substack{\sum_{i \in \bar{I}} k_{i}=t \\
0 \leq k_{i} \text { even } \leq m_{i}}}\left(\begin{array}{c}
M_{d}-k-t \\
\left.\begin{array}{c}
M_{i}-1, m_{i}-k_{i} \\
(i \in I)
\end{array}\right)
\end{array}\right) \prod_{i \in \bar{I}} \frac{B_{k_{i}}}{k_{i} !} \frac{x^{M_{d}-k-t}}{\left(M_{d}-k-t\right) !} .
$$

Using this identity, we get the following.

Proposition 1. Let d be a positive integer. Then, we obtain:

$$
\left.\Delta \phi_{m_{1}, \ldots, m_{d}}(x)=\sum_{0 \leq l \text { odd } \leq M_{d}}\left(\sum_{0 \leq k \text { odd } \leq d} \frac{1}{2^{k-1}} \sum_{\substack{|I|=k \\
0 \leq k_{i} e v e n \leq m_{i} \\
i \in \bar{I}, \sum_{i \in \bar{I}} k_{i}=l-k}} \begin{array}{c}
M_{d}-l \\
\begin{array}{c}
M_{i}-1, m_{i}-k_{i} \\
(i \in I)(i \in \bar{I})
\end{array}
\end{array}\right) \prod_{i \in \bar{I}}\left(\frac{B_{k_{i}}}{k_{i} !}\right)\right) \frac{x^{M_{d}-l}}{\left(M_{d}-l\right) !} .
$$

Using these results, we have:

Theorem 7. Let $m_{1}, \ldots, m_{d} \geq 1$, we set

$$
\phi_{m_{1}, \ldots, m_{d}}(x)=\sum_{k=0}^{M_{d}} a_{k}\left(m_{1}, \ldots, m_{d}\right) \frac{B_{k}(x)}{k !} .
$$

(a) For odd l, $1 \leq l \leq M_{d}$, we have:

$$
a_{M_{d}-l+1}\left(m_{1}, \ldots, m_{d}\right)=\sum_{0 \leq k \text { odd } \leq d} \frac{1}{2^{k-1}} \sum_{\substack{|I|=k \\
0 \leq k_{i} \text { even } \leq m_{i} \\
i \in \bar{I}, \sum_{i \in \bar{I}} k_{i}=l-k}}\left(\begin{array}{c}
M_{d}-l \\
m_{j}-1, m_{j}-k_{j} \\
(j \in I)(j \in \bar{I})
\end{array}\right) \prod_{i \in \bar{I}}\left(\frac{B_{k_{i}}}{k_{i} !}\right) .
$$

(b) For even $l, 1 \leq l \leq M_{d}$, we have $a_{M_{d}-l+1}\left(m_{1}, \ldots, m_{d}\right)=0$.

(c) We have also:

$$
\begin{gathered}
a_{0}\left(m_{1}, \ldots, m_{d}\right)= \\
\sum_{k_{d}=0}^{M_{d-1}}(-1)^{k_{d}} \sum_{k_{1}+\ldots+k_{d-1}=k_{d}}\left(\begin{array}{c}
k_{d} \\
k_{1}, \ldots, k_{d-1}
\end{array}\right) \Delta \phi_{m_{1}-k_{1}, \ldots, m_{d-1}-k_{d-1}, m_{d}+k_{d}+1}(0)
\end{gathered}
$$

with:

$$
\Delta \phi_{m_{1}-k_{1}, \ldots, m_{d-1}-k_{d-1}, m_{d}+k_{d}+1}(0)=\left(\prod_{i=1}^{d-1} \frac{B_{m_{i}-k_{i}}}{\left(m_{i}-k_{i}\right) !}\right) \frac{B_{m_{d}+k_{d}+1}}{\left(m_{d}+k_{d}+1\right) !}\left((-1)^{M_{d}+1}-1\right) .
$$


We finish the proof of Theorem 4 by using Proposition 1 and Theorem 7.

\section{Further Examples and Consequences of Theorem 4}

We restate Theorem 4 explicitly in the cases $d=2,3,4$, and we get some new recurrence formulas for Bernoulli numbers.

Example 1. For $d=2$, we have:

$$
\begin{gathered}
\phi_{m_{1}, m_{2}}(x)=(-1)^{m_{1}-1} \frac{B_{m_{1}+m_{2}}}{\left(m_{1}+m_{2}\right) !}+\sum_{k \text { even }}\left[\left(\begin{array}{c}
m_{1}+m_{2}-k-1 \\
m_{1}-k
\end{array}\right)\right. \\
\left.+\left(\begin{array}{c}
m_{1}+m_{2}-k-1 \\
m_{2}-k
\end{array}\right)\right] \frac{B_{k}}{k !} \frac{B_{m_{1}+m_{2}-k}(x)}{\left(m_{1}+m_{2}-k\right) !}
\end{gathered}
$$

We thus recover Nielsen's theorem. We give its generalized formulation in the cases $d=3,4$.

Example 2. For $d=3$, with $M_{3}=m_{1}+m_{2}+m_{3}$, we obtain:

$$
\begin{gathered}
\phi_{m_{1}, m_{2}, m_{3}}(x)=(-1)^{m_{3}-1} \sum_{a=0}^{m_{1}+m_{2}}\left[\left(\begin{array}{c}
a \\
m_{1}-1
\end{array}\right)+\left(\begin{array}{c}
a \\
m_{2}-1
\end{array}\right)\right] \frac{B_{m_{3}+a+1}}{\left(m_{3}+a+1\right) !} \cdot \frac{B_{m_{1}+m_{2}-a-1}}{\left(m_{1}+m_{2}-a-1\right) !} \\
+\frac{1}{4}\left(\begin{array}{c}
M_{3}-3 \\
m_{1}-1, m_{2}-1, m_{3}-1
\end{array}\right) \frac{B_{M_{3}-2}(x)}{\left(M_{3}-2\right) !}+\sum_{k_{1}, k_{2} \text { even }}\left[\left(\begin{array}{c}
M_{3}-k_{1}-k_{2}-1 \\
m_{1}-1, m_{2}-k_{1}, m_{3}-k_{2}
\end{array}\right)\right. \\
\left.+\left(\begin{array}{c}
M_{3}-k_{1}-k_{2}-1 \\
m_{1}-k_{1}, m_{2}-1, m_{3}-k_{2}
\end{array}\right)+\left(\begin{array}{c}
M_{3}-k_{1}-k_{2}-1 \\
m_{1}-k_{1}, m_{2}-k_{2}, m_{3}-1
\end{array}\right)\right] \frac{B_{k_{1}}}{k_{1} !} \cdot \frac{B_{k_{2}}}{k_{2} !} \cdot \frac{B_{M_{3}-k_{1}-k_{2}}(x)}{\left(M_{3}-k_{1}-k_{2}\right) !} .
\end{gathered}
$$

Example 3. For $d=4$ and $M_{4}=m_{1}+m_{2}+m_{3}+m_{4}$, we have:

$$
\begin{gathered}
\phi_{m_{1}, m_{2}, m_{3}, m_{4}}(x)=\frac{1}{4} \sum_{k \text { even }}\left[\left(\begin{array}{c}
M_{4}-k-3 \\
m_{1}-k, m_{2}-1, m_{3}-1, m_{4}-1
\end{array}\right)+\left(\begin{array}{c}
M_{4}-k-3 \\
m_{1}-1, m_{2}-k, m_{3}-1, m_{4}-1
\end{array}\right)\right. \\
\left.+\left(\begin{array}{c}
M_{4}-k-3 \\
m_{1}-1, m_{2}-1, m_{3}-k, m_{4}-1
\end{array}\right)+\left(\begin{array}{c}
M_{4}-k-3 \\
m_{1}-1, m_{2}-1, m_{3}-1, m_{4}-k
\end{array}\right)\right] \frac{B_{M_{4}-k-2}(x)}{\left(M_{4}-k-2\right) !} \cdot \frac{B_{k}}{k !} \\
+\sum_{k, k}\left[\left(\begin{array}{c}
M_{4}-k-k^{\prime}-k^{\prime \prime}-1 \\
m_{1}-1, m_{2}-k, m_{3}-k^{\prime}, m_{4}-k^{\prime \prime}
\end{array}\right)+\left(\begin{array}{c}
M_{4}-k^{\prime \prime}-1 \\
m_{1}-k, m_{2}-1, m_{3}-k^{\prime}, m_{4}-k^{\prime \prime}
\end{array}\right)\right. \\
\left.+\left(\begin{array}{c}
M_{4}-k-k^{\prime}-k^{\prime \prime}-1 \\
m_{1}-k, m_{2}-k^{\prime}, m_{3}-1, m_{4}-k^{\prime \prime}
\end{array}\right)+\left(\begin{array}{c}
M_{4}-k-k^{\prime}-k^{\prime \prime}-1 \\
m_{1}-k, m_{2}-k^{\prime}, m_{3}-k^{\prime \prime}, m_{4}-1
\end{array}\right)\right] \\
+a_{0}\left(m_{1}, m_{2}, m_{3}, m_{4}\right),
\end{gathered}
$$

where:

$$
a_{0}\left(m_{1}, m_{2}, m_{3}, m_{4}\right)=\left(1+(-1)^{M_{4}}\right) \sum_{k=0}^{M_{3}}(-1)^{k-1} \sum_{k_{1}+k_{2}+k_{3}=k}\left(\begin{array}{c}
k \\
k_{1}, k_{2}, k_{3}
\end{array}\right)\left(\prod_{i=1}^{3} \frac{B_{m_{i}-k_{i}}}{\left(m_{i}-k_{i}\right) !}\right) \frac{B_{M_{4}+k+1}}{\left(M_{4}+k+1\right) !} .
$$

From Example 1 and Example 2, with $m_{1}=m_{2}=m_{3}=m$, we have the recurrence formulas:

Corollary 3. For $m$ a positive integer greater than one, we have:

$$
\left[2\left(\begin{array}{c}
2 m-2 \\
m-1
\end{array}\right)+(-1)^{m-1}\right] \frac{B_{2 m}}{(2 m) !}=\left(\frac{B_{m}}{(m) !}\right)^{2}-2 \sum_{k=1}^{[m / 2]}\left(\begin{array}{c}
2 m-2 k-1 \\
m-2 k
\end{array}\right) \frac{B_{2 k}}{(2 k) !} \cdot \frac{B_{2 m-2 k}}{(2 m-2 k) !}
$$


and:

$$
\begin{gathered}
3\left(\begin{array}{c}
3 m-1 \\
m-1, m, m
\end{array}\right) \frac{B_{3 m}}{(3 m) !}=\frac{1}{4} \frac{(3 m-3) !}{\{(m-1) !\}^{3}} \frac{B_{3 m-2}}{(3 m-2) !} \\
+2(-1)^{m} \sum_{a=m-1}^{2 m}\left(\begin{array}{c}
a \\
m-1
\end{array}\right) \frac{B_{m+a+1}}{(m+a+1) !} \frac{B_{2 m-a-1}}{(2 m-a-1) !} \\
+3 \sum_{\substack{0 \leq k_{1}, k_{2} \text { even } \leq m \\
\left(k_{1}, k_{2}\right) \neq(0,0)}}\left(\begin{array}{c}
3 m-k_{1}-k_{2}-1 \\
m-1, m-k_{1}, m-k_{2}
\end{array}\right) \frac{B_{3 m-k_{1}-k_{2}}}{\left(3 m-k_{1}-k_{2}\right) !} .
\end{gathered}
$$

\section{Proof of Theorem 5}

It is known that:

$$
L(s, \chi)=q^{-s} \sum_{a=1}^{q} \chi(a) \zeta\left(s ; \frac{a}{q}\right)
$$

where $\zeta(s ; x)=\sum_{n \geq 0}(x+n)^{-s}$ is the Hurwitz zeta function.

Then, for $m_{1}, \ldots, m_{d}$ positive integers, with $m_{1}+\ldots+m_{d} \geq 2$, we have:

$$
L\left(-m_{i}, \chi_{i}\right)=q^{m_{i}} \sum_{k_{i}=1}^{q} \chi\left(k_{i}\right) \zeta\left(-m_{i} ; \frac{k_{i}}{q}\right)=\frac{q^{m_{i}}}{m_{i}+1} \sum_{k_{i}=1}^{q} \chi_{i}\left(k_{i}\right) B_{m_{i}+1}\left(\frac{k_{i}}{q}\right)
$$

and:

$$
\begin{gathered}
\sum_{\substack{\chi_{1} \cdots, \chi_{d}(\bmod q) \\
\chi_{1} \cdots \chi_{d}=1}} \prod_{i=1}^{d} L\left(-m_{i}, \chi_{i}\right)=\sum_{\chi_{1} \cdots \chi_{d}=1} \prod_{i=1}^{d}\left(\frac{-q^{m_{i}}}{m_{i}+1} \sum_{k_{i}=1}^{q} \chi\left(k_{i}\right) B_{m_{i}+1}\left(\frac{k_{i}}{q}\right)\right) \\
=\sum_{1 \leq k_{1}, \ldots, k_{d} \leq q}(-1)^{d} \frac{q^{M_{d}}}{\prod_{i=1}^{d}\left(m_{i}+1\right)} B_{m_{d}+1}\left(\frac{k_{d}}{q}\right)\left(\prod_{i=1}^{d-1} B_{m_{i}+1}\left(\frac{k_{i}}{q}\right)\right) \sum_{\chi_{i}} \chi_{i}\left(k_{i}\right) \overline{\chi_{i}\left(k_{d}\right)} .
\end{gathered}
$$

By use of the orthogonality relations of Dirichlet characters, we have:

$$
\sum_{\chi \bmod q} \chi(u) \overline{\chi(v)}= \begin{cases}\phi(q), & \text { if } u \equiv v \quad(\bmod q),(u v, q)=1 \\ 0, & \text { otherwise. }\end{cases}
$$

By (12), we obtain:

$$
\begin{aligned}
\sum_{\chi_{1} \cdots \chi_{d}=1} \prod_{i=1}^{d} L\left(-m_{i}, \chi_{i}\right) & =\frac{\phi(q)^{d-1} q^{M_{d}}(-1)^{d}}{\left(m_{1}+1\right) \cdots\left(m_{d}+1\right)} \sum_{\substack{(k, q) \leq 1 \\
1 \leq k \leq q}} \prod_{i=1}^{d} B_{m_{i}+1}\left(\frac{k}{q}\right) \\
& =\frac{\phi(q)^{d-1} q^{M_{d}}(-1)^{d}}{\prod_{i=1}^{d}\left(m_{i}+1\right)} \sum_{m \mid q} \mu\left(\frac{q}{m}\right) \sum_{k=1}^{m} \prod_{i=1}^{d} B_{m_{i}+1}\left(\frac{k}{m}\right) .
\end{aligned}
$$

Therefore, we have the following.

Theorem 8. If $d$ is a positive integer, then:

$$
\sum_{\chi_{1} \cdots \chi_{d}=1} \prod_{i=1}^{d} L\left(-m_{i}, \chi_{i}\right)=(-1)^{d} q^{M_{d}} \phi(q)^{d-1} m_{1} ! \cdots m_{d} ! \sum_{m \mid q} \mu\left(\frac{q}{m}\right) \sum_{k=1}^{m} \phi_{m_{1}+1, \ldots, m_{d}+1}\left(\frac{k}{m}\right) .
$$

Now, we can conclude the proof of Theorem 5 as follows. 
We write:

$$
\sum_{m \mid q} \mu\left(\frac{q}{m}\right) \sum_{k=1}^{m} \phi_{m_{1}+1, \ldots, m_{d}+1}\left(\frac{k}{m}\right)=\sum_{m \mid q} \mu\left(\frac{q}{m}\right) \sum_{k=1}^{m} \prod_{i=1}^{d} \frac{B_{m_{i}+1}(k / m)}{\left(m_{i}+1\right) !}
$$

By the relation:

$$
\phi_{m_{1}+1, \ldots, m_{d}+1}\left(\frac{k}{m}\right)=\sum_{j=0}^{M_{d}+d} a_{j}\left(m_{1}+1, \ldots, m_{d}+1\right) \frac{B_{j}(k / m)}{j !}
$$

we have:

$$
\sum_{m \mid q} \mu\left(\frac{q}{m}\right) \sum_{k=1}^{m} \phi_{m_{1}+1, \ldots, m_{d}+1}\left(\frac{k}{m}\right)=\sum_{j=0}^{M_{d}+d} \frac{a_{j}\left(m_{1}+1, \ldots, m_{d}+1\right)}{j !} \sum_{m \mid q} \mu\left(\frac{q}{m}\right) \sum_{k=1}^{m} B_{j}(k / m) .
$$

By the multiplication formula:

$$
\sum_{k^{\prime}=0}^{m-1} B_{j}\left(x+\frac{k^{\prime}}{m}\right)=m^{1-j} B_{j}(m x)
$$

we obtain:

$$
\begin{aligned}
\sum_{m \mid q} \mu\left(\frac{q}{m}\right) \sum_{k=1}^{m} \phi_{m_{1}+1, \ldots, m_{d}+1}\left(\frac{k}{m}\right) & =\sum_{j=0}^{M_{d}+d} \frac{a_{j}\left(m_{1}+1, \ldots, m_{d}+1\right)}{j !} \sum_{m \mid q} m^{1-j} \mu\left(\frac{q}{m}\right) B_{j}(1) \\
& =\sum_{j=0}^{M_{d}+d} a_{j}\left(m_{1}+1, \ldots, m_{d}+1\right) \frac{B_{j}(1)}{j !} \sum_{m \mid q} \mu\left(\frac{q}{m}\right) m^{1-j} \\
& =\sum_{j=0}^{M_{d}+d}(-1)^{j} a_{j}\left(m_{1}+1, \ldots, m_{d}+1\right) \frac{B_{j}}{j !} J_{1-j}(q) .
\end{aligned}
$$

Therefore, by Theorem 8 and the above result, we obtain:

$$
\sum_{\chi_{1} \cdots \chi_{d}=1} \prod_{i=1}^{d} L\left(-m_{i}, \chi_{i}\right)=(-1)^{d} q^{M_{d}} \phi(q)^{d-1} m_{1} ! \cdots m_{d} ! \sum_{j=0}^{M_{d}+d} a_{j}\left(m_{1}+1, \ldots, m_{d}+1\right) \frac{B_{j}}{j !} J_{1-j}(q) .
$$

This completes the proof of Theorem 5 .

\section{Conclusions}

Studies of Bernoulli numbers and polynomials have been performed in many areas. In particular, their study is used in theoretical physics, combinatorics, number theory, analytic geometry, and applied mathematics. In Theorem 7, the problem of computing Bernoulli polynomials is seen as a meaningful result of generalizing the result of Nielsen's theorem and Nörlund's formula. In summary, in Theorem 8 , we can see that the value of the $L$-function can be regarded as a meaningful result of linking Jordan functions and Dirichlet character functions using the above results.

Author Contributions: All two authors contributed equally to this work. All authors read and approved the final manuscript.

Funding: This research was supported by the Basic Science Research Program through the National Research Foundation of Korea (NRF) funded by the Ministry of Education (NRF-2018R1D1A1B07041132).

Conflicts of Interest: The authors declare no conflict of interest. 


\section{References}

1. Hecke, E. Analytische Arithmetik der Positiven Quadratischen Formen; Mathematische Werke: Copenhagen, Denmark, 1940; pp. 789-918.

2. Nielsen, N. Traité élémentaire des nombres de Bernoulli; Gauthier-Villars: Paris, France, 1923.

3. Nörlund, N.E. Vorlesungen über Differenzenrechnung; Springer: Berlin, Germany, 1924.

4. Mordell, L.J. Integral formulae of arithmetical character. J. Lond. Math. Soc. 1958, 33, 371-375. [CrossRef]

5. Mikolás, M. Integral formulae of arithmetical characteristics relating to the zeta-function of Hurwitz. Publ. Math. Debrecen 1957, 5, 44-53.

6. Zagier, D. Appendix. Curious and exotic identities for Bernoulli numbers. In Springer Monographs in Mathematics; Arakawa, T., Kaneko, M., Ibukiyama, T., Numbers, B., Functions, Z., Eds.; Springer: Berlin, Germany, 2014; pp. 239-262.

7. Carlitz, L. Note on the integral of product of several Bernoulli polynomials. J. Lond. Math. Soc. 1959, 36, 361-363. [CrossRef]

8. Hu, S.; Kim, D.; Kim, M.-S. On the integral of the product of four and more Bernoulli polynomials. Ramanujan J. 2014, 33, 281-293. [CrossRef]

9. Espinosa, O.; Moll, V.H. On some integrals involving the Hurwitz Zeta function: Part 1. Ramanujan J. 2002, 6 , 159-188. [CrossRef]

10. Espinosa, O.; Moll, V.H. On some integrals involving the Hurwitz Zeta function: Part 2. Ramanujan J. 2002, 6 , 449-468. [CrossRef]

11. Espinosa, O.; Moll, V.H. The evaluation of Tornheim double sums. Int. J. Number Theory 2006, 116, $200-229$. [CrossRef]

12. Bayad, A.; Raouj, A. Mean values of L-functions and Dedekind sums. J. Number Theory 2012, 132, $1645-1652$. [CrossRef]

(C) 2018 by the authors. Licensee MDPI, Basel, Switzerland. This article is an open access article distributed under the terms and conditions of the Creative Commons Attribution (CC BY) license (http:/ / creativecommons.org/licenses/by/4.0/). 